Bull. Austral. Math. Soc.

VoL. 55 (1997) [489-495]

\title{
AN ISOPERIMETRIC INEQUALITY FOR THE THREAD PROBLEM
}

\author{
Frank MORgan
}

\begin{abstract}
Given a fixed curve $C_{0}$ in $\mathbf{R}^{n}$ of length $L_{0}$ and a variable curve $C$ of fixed length $L \leqslant L_{0}$, the thread problem seeks a least-area surface bounded by $C_{0}+C$. We show that an extreme case is a circular arc and its chord. We provide some counterexamples and generalisations to higher dimensions.
\end{abstract}

\section{INTRODUCTION}

Given a fixed curve $C_{0}$ in $\mathbf{R}^{n}$ of length $L_{0}$ and a variable curve $C$ of fixed length $L \leqslant L_{0}$, the classical thread problem seeks a least-area surface bounded by $C_{0}+C$. The desired isoperimetric inequality for the least area $A$ should take the scale-invariant form

$$
A \leqslant L_{0}^{2} f\left(L / L_{0}\right)
$$

for a continuous function $f$ which vanishes when $L=L_{0}$ The classical isoperimetric inequality says only that $A \leqslant\left(L_{0}+L\right)^{2} / 4 \pi=L_{0}^{2}\left(1+L / L_{0}\right)^{2} / 4 \pi$, which fails to vanish when $L=L_{0}$. Theorem 2.3 provides a sharp isoperimetric inequality by proving the extreme case to be a circular arc of arc length $L_{0}$ and chordal distance $L$.

If the curve $C_{0}$ is allowed several components, no isoperimetric inequality of form (1) holds (see Section 2.4).

The analogous question in higher dimensions (see Ecker [5]) replaces curves and discs by the rectifiable currents (generalised $k$-dimensional oriented surfaces) of geometric measure theory (see [6]). Theorem 3.1 shows that if the fixed boundary surface $C_{0}$ is closed, the extreme case is two concentric spheres. Without the closure hypothesis, no general isoperimetric inequality holds (see Section 3.2).

For more information on the thread problem see Dierkes, Hildebrandt, Küster, and Wohlrab [4, Chapter 10] and Nitsche [7].

Received 3 July 1996

I would like to thank Klaus Ecker for posing the problem and for his hospitality on my visit to Melbourne University in February, 1995. Mike Gage, Bob Connelly, and Charlie Epstein identified Proposition 2.2 as a special case of Schur's Lemma and provided references. The final inequality in Theorem 2.3 is due to two Bennington College undergraduate students of Professor Jerry Bope: Wenhuan Zhao and Jing Lu. This work was partially supported by a National Science Foundation grant.

Copyright Clearance Centre, Inc. Serial-fee code: 0004-9729/97 \$A2.00+0.00. 


\section{AN ISOPERIMETRIC INEQUALITY FOR THE THREAD PROBLEM FOR CURVES IN $\mathbf{R}^{n}$}

Theorem 2.3 provides our main isoperimetric inequality for the thread problem for curves in $\mathbf{R}^{n}$. It depends on an extremal property of circular arcs, Proposition 2.2, a special case of Schur's Lemma (see [3, p.36]), which follows easily from Lemma 2.1 .

LEMMA 2.1. For $0<a<\pi$, consider a map

$$
\gamma:[-a, a] \rightarrow S^{n-1}
$$

mapping 0 to the south pole, of Lipschitz constant 1. Then $\left|\int \gamma\right|$ is uniquely minimised when $\gamma$ is an arc of a great circle.

Proof: Let $\int \gamma=\left(a_{1}, \ldots, a_{n}\right)$. Then $a_{n}<0$, and $a_{n}$ is maximised by an arc of a great circle, for which $a_{1}=\ldots=a_{n-1}=0$. Conversely if $a_{n}$ is maximised, $\gamma$ must consist of two arcs of great circles; if further $a_{1}=\ldots=a_{n-1}=0$, together they form an arc of a single great circle.

PROPOSITION 2.2. A circular arc in $\mathbf{R}^{n}$ uniquely minimises the distance between its endpoints among all curves with the same length and no greater curvature.

(A differentiable curve $\gamma$ parametrised by arc length has curvature less than or equal to $\kappa$ if $\left|\dot{\gamma}\left(t_{2}\right)-\dot{\gamma}\left(t_{1}\right)\right| \leqslant \kappa\left|t_{2}-t_{1}\right|$.)

Proof: Apply Lemma 2.1 to $\dot{\gamma}$.

THEOREM 2.3. Let $C_{0}:\left[0, L_{0}\right] \rightarrow \mathbf{R}^{n}$ be a rectifiable curve parametrised by arc length. Choose another such $C$ of prescribed length $L$,

$$
\left|C_{0}\left(L_{0}\right)-C_{0}(0)\right| \leqslant L \leqslant L_{0},
$$

to minimise the area $A$ of an area-minimising disc $D$ bounded by $C_{0}+C$. Then $A$ is less than or equal to the area $A_{0}$ bounded by a circular arc of length $L_{0}$ and its chord of length $L$ :

$$
A \leqslant A_{0} \leqslant L_{0}^{2} \sqrt{1-L / L_{0}} / \sqrt{6} .
$$

The final inequality is asymptotically sharp as $L / L_{0} \rightarrow 1$.

The square root expresses the fact that varying a straight line sweeps out area to first order but changes length only to second order.

REMARK. A variational argument shows that $C$ is a $C^{1,1}$ curve, with constant curvature away from $C_{0}$ and no greater curvature along $C_{0}$, unless it is completely contained in $C_{0}$.

Proof: We shall actually show that given $C_{0}$ of length $L_{0}$ and

$$
\left|C_{0}\left(L_{0}\right)-C_{0}(0)\right| \leqslant L_{1} \leqslant L_{0}
$$


for all $L_{1} \leqslant L \leqslant L_{0}, A$ is less than or equal to the area bounded by circular arcs of lengths $L_{0}, L$ with common chord of length $L_{1}$. The special case $L=L_{1}$ proves the theorem.

If $L=L_{0}$, then the conclusion holds trivially with $C=C_{0}$ and $D=\emptyset$. Compactness arguments shows that the set of $L$ for which the conclusion holds is closed. Therefore it suffices to show that if the conclusion holds for any $L>L_{1}$, it holds for slightly smaller $L$.

If $C$ is not a circular arc, then its curvature is not weakly bounded by that of a circular arc of length $L$ and chordal length $L_{1}$ by Proposition 2.2 , because $L_{1} \leqslant$ $\left|C_{0}\left(L_{0}\right)-C_{0}(0)\right|$. Consequently, whether or not $C$ is a circular arc, there are small smooth variations of $C$ reducing length by $\varepsilon$ and sweeping out a piece of surface $S_{\varepsilon}$ of area no greater than the area between circular arcs of lengths $L$ and $L-\varepsilon$.

To verify the final inequality, note that in terms of the radian measure $\theta$ and radius of curvature $r$ of the arc, its arc length $L_{0}=r \theta$, its chord length $L=2 r \sin (\theta / 2)$, and its area $A_{0}=(\theta-\sin \theta) r^{2} / 2$. (Since $A_{0} / L_{0}^{2} \sim \theta / 12$ and $1-L / L_{0} \sim \theta^{2} / 24$, therefore the inequality is asymptotically sharp.) After squaring both sides, the desired inequality becomes

$$
f(\theta)=\theta^{4}+3 \theta \sin \theta-(3 / 2) \theta^{2}-(3 / 2) \sin ^{2} \theta-2 \theta^{3} \sin (\theta / 2) \geqslant 0 .
$$

The estimates

(1) $\sin \theta \geqslant \theta-\theta^{3} / 3$ !,

(2) $\sin ^{2} \theta \leqslant \theta^{2}$, and

(3) $\sin (\theta / 2) \leqslant \theta / 2-\theta^{3} / 2^{3} 3 !+\theta^{5} / 2^{5} 5$ !

imply that

$$
f(\theta) \geqslant\left(\theta^{4} / 2^{4} 5 !\right)\left(-\theta^{4}+80 \theta^{2}-960\right) \geqslant 0
$$

if $\theta^{2} \geqslant 40-8 \sqrt{10} \approx 14.7$. The estimates

(1.') $\sin \theta \geqslant \theta-\theta^{3} / 3 !+\theta^{5} / 5 !-\theta^{7} / 7$ !,

$\left(2^{\prime}\right) \quad \sin ^{2} \theta \leqslant \theta^{2}-2^{3} \theta^{4} / 4 !+2^{5} \theta^{6} / 6 !-2^{7} \theta^{8} / 8 !+2^{9} \theta^{10} / 10$ !

and (3) imply that

$$
f(\theta) \geqslant \theta^{8}\left(a-b \theta^{2}\right) \geqslant 0,
$$

with

$$
\begin{aligned}
& a=(3 / 2)\left(2^{7} / 8 !\right)-3 / 7 !-2 / 2^{5} 5 ! \approx .00365 \\
& b=(3 / 2)\left(2^{9} / 10 !\right) \approx .000212
\end{aligned}
$$

if $\theta^{2} \leqslant a / b \approx 17.2$. 
2.4 SEveral COMPONENTS: If $C_{0}$ and $C$ are allowed several components, the least area need not even approach 0 as $L \rightarrow L_{0}$. Let $C_{0}$ be the top and bottom of a rectangle of length 1 and height $1-\varepsilon$ and take $L=2$. Then $C$ must be the two sides and the enclosed area equals $1-\varepsilon$, which does not go to 0 as $\varepsilon \rightarrow 0$.

One can, however, allow $C_{0}$ and $C$ to have additional closed curves and allow surfaces of higher genus. The same proof applies.

\section{AN ISOPERIMETRIC INEQUALITY FOR THE THREAD PROBLEM FOR CLOSED SURFACES IN GENERAL DIMENSIONS}

Theorem 3.1 provides an isoperimetric inequality for the thread problem for closed $k$-dimensional surfaces in $\mathbf{R}^{n}$. As surfaces we use the oriented rectifiable currents of geometric measure theory (see [6]), for which length or area (counting multiplicity) is called mass $\mathbf{M}$.

THEOREM 3.1. Let $C_{0}$ be a $k$-dimensional rectifiable current without boundary in $\mathbf{R}^{n}$. Choose another such $C$ of prescribed mass

$$
0 \leqslant \mathrm{M}(C) \leqslant \mathrm{M}\left(C_{0}\right)
$$

to minimise the mass of a $(k+1)$-dimensional mass-minimising rectifiable current $S$ bounded by $C_{0}+C$. Then

$$
\mathbf{M}(S) \leqslant \gamma_{k+1}\left(\mathbf{M}\left(C_{0}\right)^{(k+1) / k}-\mathbf{M}(C)^{(k+1) / k}\right),
$$

where $\gamma_{k+1}$ is the optimal isoperimetric constant

$$
\gamma_{k+1}=\frac{\operatorname{vol} \mathbf{B}^{k+1}(1)}{\left(\operatorname{area} \mathbf{S}^{k}(1)\right)^{(k+1) / k}}
$$

REMARK. The existence of such $C$ and $S$ comes from compactness arguments of geometric measure theory $[6,5.5]$. (First, for given $C$, there is a minimiser $S$. Second, choose $C$ with mass less than or equal to the prescribed mass $M_{0}$ to minimise $\mathbf{M}(S)$. Third argue that unless $\mathrm{M}(C)=M_{0}, \mathrm{M}(S)$ could be further reduced.)

A variational argument shows that $C$ has weakly bounded mean curvature (unless it is contained in $C_{0}$ ) and hence is a $C^{1}$ embedded manifold on an open dense set [1, Section 8]. If $k=n-1$, away from $C_{0}, C$ is a $C^{\infty}$ embedded constant-mean-curvature manifold (possibly with multiplicity) except for a singular set of dimension at most $n-8$ $[6,8.6]$.

Proof: If the prescribed mass $M=\mathrm{M}\left(C_{0}\right)$, then (1) holds trivially with $C=C_{0}$ and $S=0$. Compactness arguments show that the set of $M$. for which (1) holds is 
closed. Therefore it suffices to show that if (1) holds for any $M$, it holds for slightly smaller $M$.

If $C$ is not a round sphere, then its mean curvature is not weakly bounded by the mean curvature of a round sphere of area $M[2,(3)$, p.452]. Consequently, whether or not $C$ is a round sphere, there are small smooth variations of $C$ reducing mass by $\varepsilon$ and sweeping out a piece of $(k+1)$-dimensional surface $S_{\varepsilon}$ of no greater mass than the difference of the volumes of round spheres of area $M$ and $M-\varepsilon$ :

$$
\mathbf{M}\left(S_{\varepsilon}\right) \leqslant \gamma_{k+1}\left(M^{(k+1) / k}-(M-\varepsilon)^{(k+1) / k}\right) .
$$

Therefore $S+S_{\varepsilon}$ satisfies (1).

3.2. NonClosed SURfaCes. If the hypothesis that $C$ be closed is omitted from Theorem 3.1, no isoperimetric inequality holds. For example, let $C_{0}$ be a portion of a catenoid of area $A$ between two parallel congruent circles which bound discs of total area $A-\varepsilon$. As $\varepsilon$ approaches 0 , the volume enclosed does not approach 0 . The boundary circles may be connected by a thin bridge.

\section{A Singular EXAMPLE}

Example 4.1 (Case b, $L=L_{0} / 2 \leqslant 4 \pi$ ) shows that the thread need not be $C^{1,1}$, even at a point on the support of the area-minimising surface. (The thread is contained in the fixed boundary curve.) We are allowing general oriented threads and surfaces of any number of componments (rectifiable currents).

Example 4.1. Let $C_{0}$ be a $C^{1}$ "Figure 8 " in $\mathbf{R}^{2}$, consisting of two crossing line segments and two unit circular arcs, of total length $L_{0}$, as in Figure 1. For $0 \leqslant L \leqslant L_{0}$, consider a thread (1-dimensional rectifiable current) $C$ of length $L$ which minimises the area bounded by $C_{0}+C$. Then $C$ falls into one of the four cases $a-d$ of Figure 2, consisting of circular arcs of the same curvature and portions of $C_{0}$. Each case occurs for some $C_{0}, L$, as indicated in Figure 2 .

Proof: $C_{0}$ and $C$ are boundaries of oriented regions (rectifiable currents) $R_{0}$, $R$. The multiplicity of $R_{0}$ is always \pm 1 . We claim that the multiplicity of $R$ is always \pm 1 . Otherwise, changing every positive multiplicity of $R$ to +1 and every negative multiplicity to -1 would reduce the length $\mathbf{M}(C)$ and the area $\mathbf{M}\left(R_{0}+R\right)$; but $C$ minimises $\mathrm{M}\left(R_{0}+R\right)$ among curves with length at most $L$. Therefore $R=R_{1}-R_{-1}$, where $R_{1}$ is the region of multiplicity 1 and $R_{-1}$ is the region of multiplicity -1 .

Now we note that $R_{1}$ is contained in the top half of the Figure 8 . Otherwise, replacing $R_{1}$ by its restriction to the top half would reduce $M\left(R_{0}+R\right)$ without increasing the length of $C$, a contradiction. Similarly, $R_{-1}$ is contained in the bottom half of the Figure 8. 


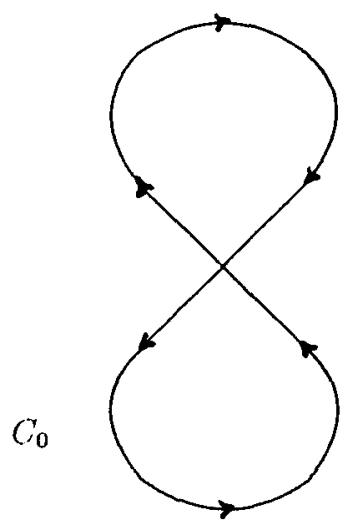

Figure 1 The fixed boundary curve $C_{0}$ is a "Figure $8 . "$

By a variational argument, away from the vertex, $\partial R$ is a $C^{1,1}$ curve consisting of circular arcs, all of the same curvature, and pieces of $C_{0}$, of no greater curvature. If it contained two circles, expanding one and shrinking the other could maintain area and reduce length (unless neither could be expanded as in degenerate Case c. Therefore, all possibilities are represented by Cases a-d.

It remains to be shown that each case occurs as claimed. Case a must occur if $L \leqslant 2 \pi$, because the Cases b-d have $L>2 \pi$. Similarly Cases c, d must occur as claimed. We now show that Case b occurs if $2 \pi<L \leqslant \min \left\{L_{0} / 2,4 \pi\right\}$ for the equivalent problem of minimising $L$ for fixed area $A \leqslant A_{0}$, where $A_{0}$ is the area of half the Figure 8. We must rule out Case c. Consider generalisations of Case c, in which the circular arc has radius of curvature $r_{1}$ and the circle has radius $r_{2}$, so that the upper area is of the form $B_{0} r_{1}^{2}$ and the lower area equals $A_{0}-\pi r_{2}^{2}$. For a minimiser, $r_{1}=r_{2} \equiv r_{0} ;$ since $B_{0} r_{0}^{2}+A_{0}-\pi r_{0}^{2} \leqslant A_{0}$, therefore $B_{0} \leqslant \pi$. If we decrease $r_{1}$ and $r_{2}$ keeping area fixed, $r_{1} \geqslant r_{2}$. Hence the rate of increase of length $\partial R_{1}$ is less than the rate of decrease of length $\partial R_{-1}$ and total length decreases as we approach Case $b$, which must therefore provide the minimiser.

\section{REFERENCES}

[1] W.K. Allard, 'On the first variation of a varifold', Ann. of Math. 95 (1972), 417-491.

[2] F.J. Almgren, 'Optimal isoperimetric inequalities', Indiana Univ. Math. J. 35 (1986), 451-547.

[3] S.S. Chern, 'Curves and surfaces in Euclidean space', in Studies in Global Geometry and Analysis, (S.S. Chern, Editor), Studies in Mathematics 4 (Math. Assn. of Amer., 1967), pp. 16-56.

[4] U. Dierkes, S.. Hildebrandt, A. Küster and O. Wohlrab, Minimal surfaces II: Boundary regularity (Springer-Verlag, Berlin, Heidelberg, New York, 1992). 


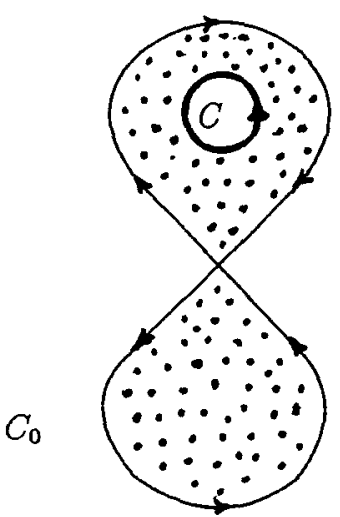

Case a

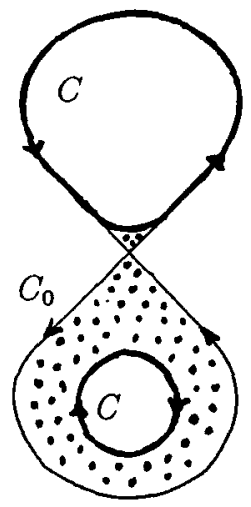

Case c

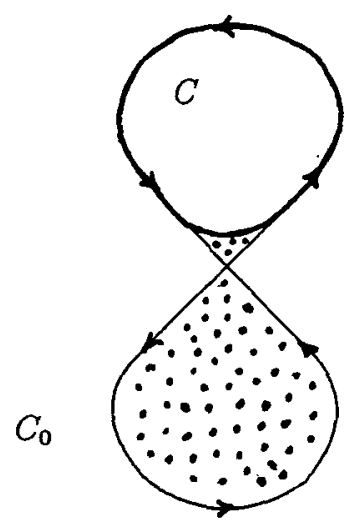

Case b

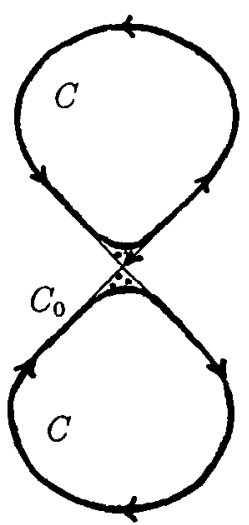

Case d

Figure 2 The solution to the thread problem falls into four cases, all of which occur.

[5] K. Ecker, 'Area-minimizing integral currents with movable boundary parts of prescribed mass', Ann. Inst. H. Poincaré 6 (1989), 261-293.

[6] F. Morgan, Geometric measure theory: a beginner's guide, (second edition) (Academic Press, New York, 1995).

[7] J.C.C. Nitsche, Vorlesungen über Minimalfï̈chen (Springer-Verlag, Berlin, Heidelberg, New York, 1975).

\section{Department of Mathematics}

Williams College

Williamstown, MA 01267

United States of America 